
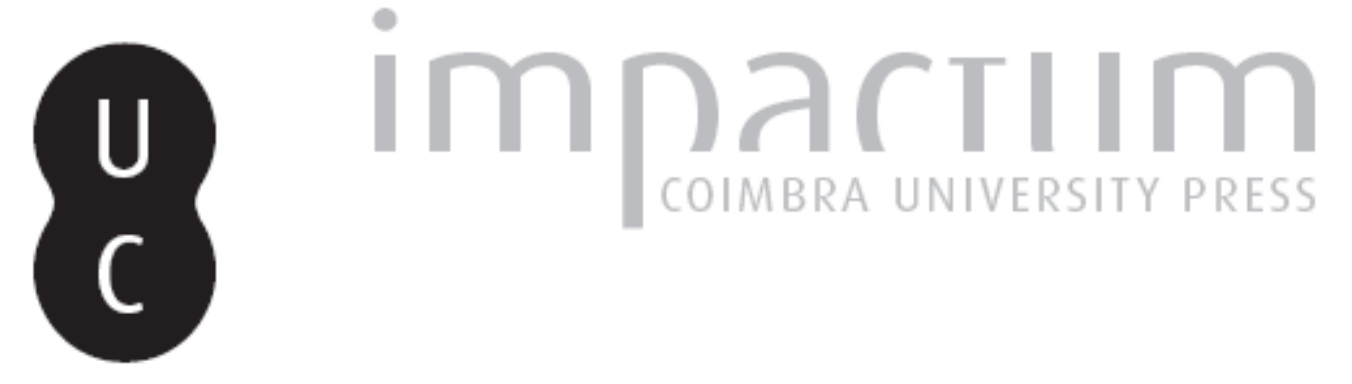

\title{
La Traviata di Lisbona: note quasi autobiografiche attorno a un bicentenario
}

Autor(es): Beghelli, Marco

Publicado por: Imprensa da Universidade de Coimbra

URL persistente:

URl:http://hdl.handle.net/10316.2/42428

DOI:

DOI:https://doi.org/10.14195/0870-8584_8_4

Accessed : $\quad$ 26-Apr-2023 12:41:53

A navegação consulta e descarregamento dos títulos inseridos nas Bibliotecas Digitais UC Digitalis, UC Pombalina e UC Impactum, pressupõem a aceitação plena e sem reservas dos Termos e Condições de Uso destas Bibliotecas Digitais, disponíveis em https://digitalis.uc.pt/pt-pt/termos.

Conforme exposto nos referidos Termos e Condições de Uso, o descarregamento de títulos de acesso restrito requer uma licença válida de autorização devendo o utilizador aceder ao(s) documento(s) a partir de um endereço de IP da instituição detentora da supramencionada licença.

Ao utilizador é apenas permitido o descarregamento para uso pessoal, pelo que o emprego do(s) título(s) descarregado(s) para outro fim, designadamente comercial, carece de autorização do respetivo autor ou editor da obra.

Na medida em que todas as obras da UC Digitalis se encontram protegidas pelo Código do Direito de Autor e Direitos Conexos e demais legislação aplicável, toda a cópia, parcial ou total, deste documento, nos casos em que é legalmente admitida, deverá conter ou fazer-se acompanhar por este aviso.

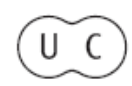




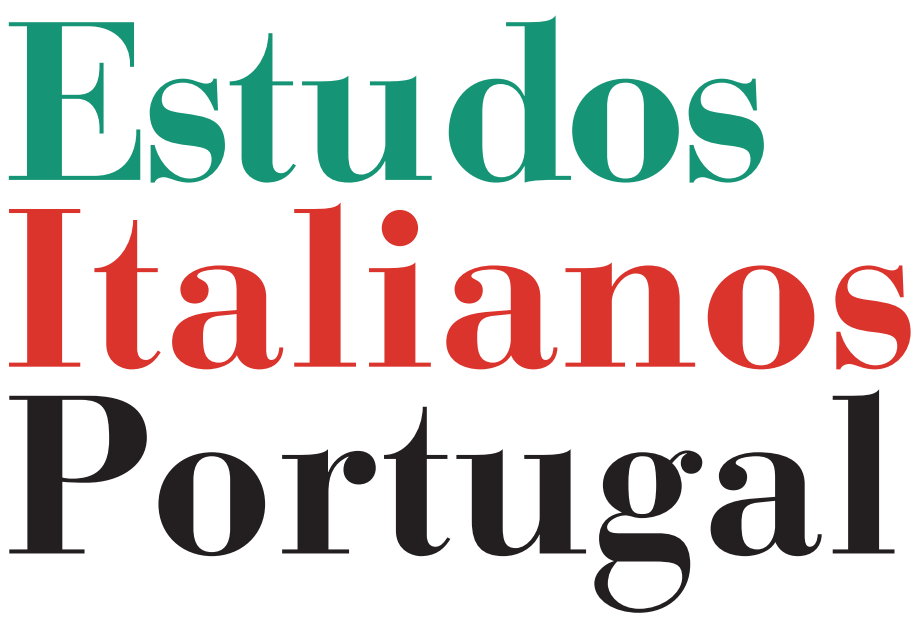

Instituto

Italiano

de Cultura

de Lisboa

Nova Série

$\mathbf{N}^{\circ} \mathbf{8}$ 


\section{LA TRAVIATA DI LISBONA: NOTE QUASI AUTOBIOGRAFICHE ATTORNO A UN BICENTENARIO}

Marco Beghelli*

AnNo 2013: bicentenario della nascita di Giuseppe Verdi (1813-1901). Fra le tante iniziative celebrative susseguitesi nel mondo, il Teatro Nacional de São Carlos si segnala per aver voluto rappresentare a Lisbona in rapida alternanza i tre capolavori del Verdi maturo: Rigoletto (1851), Il trovatore (1853), La traviata (1853), direttore Martin André, regista Francesco Esposito. Sin dalla sua fondazione (1793), il Teatro de São Carlos è stato legato a filo doppio all'opera italiana, non solo per aver ospitato i titoli maggiori del repertorio, ma soprattutto per aver chiamato sul suo palcoscenico un grande numero di cantanti di scuola italiana che consideravano Lisbona una tappa importante nella loro carriera. E fra le opere allestite più di frequente negli ultimi 150 primeggiano proprio quelle di Verdi, come mise ben in luce l'esposizione Verdi em Portugal, 1843-2001, commemorativa del primo centenario della morte del compositore, allestita a Lisbona nella Biblioteca Nacional de Portugal (luglio 2001).

* Docente nell'Università di Bologna, dedica le sue ricerche al teatro d'opera fra Sette e Novecento, affrontato da diverse prospettive (storica, drammaturgica, sociologica, semiotica, filologica). Ha fondato l'Archivio del Canto, dove si raccolgono libri e fondi documentari relativi a cantanti del passato (http://www.muspe.unibo.it/archivio-del-canto). Il suo libro più recente: Ermafrodite armoniche: il contralto nell'Ottocento (con Raffaele Talmelli). 
Per chi abbia a cuore la diffusione della cultura italiana nel mondo, ogni anniversario verdiano non rappresenta però solo il festeggiamento di un musicista italiano di prima grandezza, ma di uno fra $\mathrm{i}$ simboli più tangibili con cui l'Italia viene da tempo identificata sulla superficie dell'intero pianeta. In tal senso, la figura di Giuseppe Verdi finisce per surclassare quella di altri personaggi anche politicamente e culturalmente più importanti per la nazione italiana, ma non necessariamente per il mondo.

Sul piano strettamente politico, è vero che l'Italia non vanta figure di forte impatto sovranazionale quali un Carlo Magno, un Napoleone o un Kennedy, e sui libri di scuola italiani l'importanza strategica di personaggi quali Lorenzo il Magnifico o Vittorio Emanuele II viene evidenziata certamente più di quanto accada all'estero. Diverso è il discorso sul piano artistico in senso lato, con innumerevoli esponenti italiani di spicco dal Medioevo ai nostri giorni, riconosciuti e apprezzati in tutto il mondo; ma è ben chiaro che, per quanto possa essere universalmente noto l'operato di Michelangelo attraverso i libri e i film documentari, soltanto giungendo materialmente in Italia sarà possibile farsi una reale idea del suo Giudizio Universale, mentre la potenza della Commedia dantesca resta inevitabilmente preclusa a chi non goda di una perfetta padronanza della lingua in cui è scritta.

La musica di Verdi, invece (ma è caratteristica di tutta la musica), non esiste sulla carta, come esistono il verso del poeta o lo schizzo del disegnatore: ciò che i segni graficomusicali hanno in rapporto col suono fisicamente udibile è un legame prescrittivo di tipo simbolico (a segno corrisponde suono), né più né meno di quanto avviene con la stringa di numeri contenuti in un file informatico in rapporto all'immagine digitale che si produce a beneficio degli occhi (a numero corrisponde colore). Ne consegue che, per godere di quella fetta di cultura italiana che vive sotto il nome di Giuseppe Verdi, non è necessario spostarsi in Italia (come 
per vedere Michelangelo) o studiare la lingua italiana antica (come per leggere Dante), ma è la musica a girare il mondo in cerca dell'ascoltatore, e si rinnova di volta in volta in sua presenza, proponendosi sic et simpliciter alle sue orecchie.

Certo, il discorso vale anche per Vivaldi, Rossini o Puccini, ma nessuno di questi pur sommi musicisti sembra potersi dire portatore di italianità nel mondo quanto Verdi. Non è un caso che, fino a quando sussistevano le valute nazionali a caratterizzare il sistema monetario di ogni singolo paese europeo, il primo impatto che il turista aveva nel secolo scorso con la nuova Italia repubblicana, prima ancora di mettervi piede, per il solo atto di acquistare preventivamente in banca lire italiane, era proprio con Giuseppe Verdi, il cui volto campeggiava sulle banconote più diffuse. E quando negli anni '70 la Zecca rinnovò l'intero parco della cartamoneta, fu deciso di cambiare il ritratto identificativo per tutti i tagli tranne che per le Mille Lire, che vennero confermate appannaggio di Giuseppe Verdi, pur sottoposto a restyling integrale.

Ma Verdi è naturalmente in primo luogo musica. Temi melodici come quelli di "Libiamo ne' lieti calici" (da La traviata) o "La donna è mobile" (da Rigoletto) sono assurti fra i primi a hit da suoneria di telefono cellulare, non foss'altro per l'icastica semplicità delle rispettive melodie, facilmente memorizzabili e riproducibili anche dai sistemi MIDI meno sofisticati. Nulla di nuovo: durante le prove dell'opera Rigoletto, andata in prima esecuzione al Teatro La Fenice di Venezia nel 1851, Verdi vietò al tenore di turno di provare pubblicamente "La donna è mobile" fino all'ultimo momento, temendo che l'orecchiabilità del tema udito da coristi, sarti e macchinisti avrebbe favorito una sua precoce diffusione al di fuori del teatro, passando anzitempo di bocca in bocca, e guastando così l'effetto sorpresa nella sera del debutto.

Tutto ciò per sottolineare che Verdi non aveva certo bisogno di un atteggiamento protezionistico per giungere al maggiore successo. Eppure una sorta di "protezione politi- 
ca” vi fu, ed astutamente efficace, onde elevare la figura del popolare artista al di sopra del mondo della musica, in un più sacro empireo nazionale. L'Italia che nel 1861 raggiunge dopo secoli la sua unità territoriale, aveva bisogno innanzitutto di "fare gli Italiani”, diceva Massimo d'Azeglio. E per raggiungere una unità di popolo erano necessarie - come altrove - unità di lingua, di religione e di cultura. Nessun problema per l'unità di religione, almeno nei primi 100 anni di vita della nuova nazione (la mancanza d'unità religiosa è semmai una realtà di oggi). Per l'unità di lingua è noto come sarà necessario attendere la televisione, a metà del secolo XX. L'unità di cultura si poteva invece costruire artificialmente sin da subito, attraverso una serie di operazioni mirate. Era ad esempio necessario ufficializzare un drappello di padri della patria, figure di riferimento che avevano contribuito a costruire la nuova Italia, da additare alle nuove generazioni come eroi fondatori: Garibaldi, Mazzini, Cavour, Verdi. E Verdi, padre artistico, venne subito caricato anche di una connotazione politica superiore all'effettiva realtà dei fatti, culminante con la sua nomina a senatore del Regno (1874).

Nacque ad esempio in quei primi anni di unità nazionale il mito del $V a$, pensiero, sull'ali dorate, il famoso coro al centro dell'opera Nabucco: si disse che sin dalla prima recita, a Milano nel 1842, il canto appassionato degli ebrei che sognano la loro "patria sì bella e perduta" era stato bissato a furor di popolo, trovandosi da subito in Verdi e in quella musica un paladino delle aspirazioni risorgimentali. Oggi sappiamo che il coro effettivamente bissato alla prima esecuzione non fu quello patriottico, bensì quello religioso (il coro finale "Immenso Geova"); ma l'immagine di un Verdi patriota fin dalla prima ora faceva buon gioco agli artefici dell'Italia unita, ed è proseguita fino ai giorni nostri: quanti libri propagandistici per l'infanzia hanno riassunto visivamente la storia del Risorgimento italiano affiancando in appositi quadretti cinti d'alloro l'ideologo Giuseppe Mazzini, lo statista Camillo Benso 
conte di Cavour, il generale Giuseppe Garibaldi e il bardo Giuseppe Verdi!

La fama artistica di Verdi fra i contemporanei non è paragonabile a quella di Rossini, significativamente appellato da Stendhal il "Napoleone della musica", colui che a 25 anni aveva già fatto piazza pulita d'ogni rivale nei teatri d'Europa così come Bonaparte aveva rovesciato i governi d'ogni paese. Verdi scrive Rigoletto, Il trovatore, La traviata alla soglia dei 40 anni di età; se fosse morto prima (come Mozart e Pergolesi, Bellini e Chopin, Mendelssohn e Schubert), l'Istituto italiano di cultura non mi avrebbe invitato a Lisbona in quest'anno 2013 per celebrarne i fasti e non starei ora a scrivere queste poche righe commemorative in piena estate, giacché quasi tutto quanto Verdi ebbe a scrivere prima di quell'età (ben 16 opere!) non sarebbe stato sufficiente a renderlo immortale. Ma lo stesso discorso varrebbe in gran parte anche per Wagner, l'altro dioscuro del teatro melodrammatico di cui si festeggia quest'anno il bicentenario della nascita.

Dalla metà del secolo, la stella verdiana brillò tuttavia imperitura, e senza alcun bisogno di artificiosi sostegni politici che, come già detto, giunsero a cose fatte, appropriandosi opportunisticamente di una fama già consolidata. A sostenere Verdi e le sue opere di fronte al pubblico furono piuttosto i cantanti, che adattarono le proprie caratteristiche vocali alle nuove esigenze della scrittura verdiana, così essenziale e diretta rispetto a quella idealizzata, quasi "tardo barocca", di Rossini. Durante gli ultimi anni di vita di Verdi, fra le mille prodezze della tecnica, prese corpo anche l'opportunità della registrazione sonora. All'alba del secolo XX si diffonde dunque a macchia d'olio quel nuovo strumento di divulgazione musicale che è il disco; e fra i primi dischi a 78 giri le arie di Verdi sono i brani più registrati in assoluto dai cantanti dell'epoca, i quali le preferiscono ai compositori del momento (Puccini, Mascagni, Leoncavallo, Giordano, Cilea, Massenet, Strauss). 
Attorno ai cantanti, per la prima volta immortalati dal disco e dunque fruibili anche in absentia, cresceranno con rapidità $\mathrm{i}$ ben noti fenomeni di melomania operistica che contraddistinguono l'ambiente. Nell'immaginario collettivo (anche per la mancanza di importanti opere nuove, atte a sostituire quelle divenute ormai di repertorio), si tenderà così sempre più spesso a parlare dell' Aida "di Caruso", dell'Otello "di Del Monaco", della Traviata "della Callas" - piuttosto che "di Verdi". L'identificazione fra personaggio e interprete travalica l'autore, e l'opera assurge al rango di un sacro rito per l'esibizione di vestali canore che sera dopo sera si immolano su quell'ara sacrale chiamata palcoscenico.

Nel 1958, Lisbona fu per pochi giorni protagonista assoluta di uno di tali fenomeni di delirio melomaniacale: al Teatro Nacional de São Carlos si dava La traviata di Giuseppe Verdi. La cosa non era di per sé una notizia: La traviata si era ormai rappresentata decine di volte a Lisbona nei precedenti cento e più anni, e si eseguiva praticamente ogni settimana in qualche teatro del mondo, grande o piccolo che fosse. L'interesse non era dato dunque dalla Traviata in sé, ma dalla presenza nella parte di protagonista del soprano Maria Callas (1923-1977), che nel 1958 era già in odore di santificazione artistica.

L'evento fu preparato con tutti i crismi, ma le trattative non erano state semplici. La prima intervista rilasciata dalla Callas, ancora in aeroporto (25 marzo 1958), fa credere che la proposta di cantare La traviata fosse venuta dal Teatro, accettata di buon grado dall'artista; gli scambi epistolari superstiti rivelano invece che la "Divina" si era imposta strenuamente perché il debutto a Lisbona avvenisse proprio in quel ruolo a lei particolarmente congeniale.

Se il personaggio di Violetta si identifica in maniera naturale con l'arte di Maria Callas, è altrettanto vero che l'artista vi si dedicò in un periodo relativamente ristretto della sua carriera, soltanto fra il 1951 e il 1958. La Traviata di Lisbona 
(due recite quasi senza prove: 27 e 30 marzo 1958) fu dunque fra le ultime apparizioni della Callas in quell'opera (nelle settimane successive ancora a Londra e infine a Dallas). Fu un successo, secondo previsione. Ma poi l'evento scivolò verso l'oblio, surclassato da altri eventi artistici e mondani più eclatanti.

Non se ne parlò più per parecchi anni, fino a quando un nuovo mito canoro, questa volta maschile, non cominciò a prendere corpo nel cuore dei melomani più incalliti: quello del tenore spagnolo Alfredo Kraus (1927-1999). Di soli quattro anni più giovane della Callas, Kraus faticò non poco a trovare un suo spazio nell'agone lirico di quegli anni '50'60, per l'affidarsi a una vocalità stilisticamente troppo antica (si rifaceva a stilemi preverdiani) e troppo moderna insieme (rifuggì sempre il canto appassionatamente verista dei suoi colleghi tenori di quegli anni). Soltanto a partire dagli ultimi anni '70 la diversa sensibilità degli spettatori di nuova generazione gli riconobbe progressivamente i giusti meriti. Venire dunque a sapere dagli annali teatrali che nel lontano 1958 Callas e Kraus avevano per due sere cantato insieme, l'una al culmine della popolarità artistica e presto anche scandalistica, l'altro praticamente sconosciuto e messo in quanto tale a fianco della star per non farla sfigurare, scatenò negli appassionati cultori delle registrazioni dal vivo "non ufficiali" un desiderio forse inappagabile di poter ascoltare un giorno le due voci amate intrecciarsi nel canto di "Parigi, o cara, noi lasceremo" e sentire la "Divina" invocare nell'"Amami, Alfredo" il nome personale dell'ormai idolatrato tenore.

Furono le ricerche attente della signora Kraus a far saltar fuori dagli archivi della radio portoghese i nastri dimenticati di quella esecuzione a suo tempo registrata e trasmessa via etere. I dischi immediatamente prodotti totalizzarono un boom di vendite fra i fanatici collezionisti, e La traviata di Lisbona, come venne subito battezzata completando ad hoc l'originario titolo verdiano, venne riconosciuta come la 
migliore registrazione della Traviata di Maria Callas, la quale per un vincolo di copyright non aveva potuto registrare l'opera ufficialmente negli studi della EMI - La Voce del Padrone, al pari delle altre del suo vasto repertorio.

Un'idea del feticismo che si produsse attorno a tale registrazione in quegli anni '80, soprattutto negli ambienti omosessuali particolarmente legati al culto di Callas e Kraus, si può avere dalla commedia The Lisbon Traviata scritta nel 1989 da un autore del calibro di Terrence McNally (classe 1938), dove i difficili rapporti fra coppie gay in crisi vengono dipanati sullo sfondo di lunghi e attenti ascolti consolatori di quella "registrazione feticcio" del melodramma più melodrammatico, fra sogno e realtà. Da quel momento, il nome di Lisbona evoca nei melomani di tutto il mondo l'associazione con La traviata, con quella particolare Traviata: la Traviata di Lisbona per antonomasia, evocata in città a cinquant'anni di distanza da una memorabile esposizione al Museu da Electricidade (luglio-ottobre 2008), organizzata con il patrocinio dell'Associazione Internazionale "Maria Callas".

Invitato a Lisbona nella primavera di questo 2013 per celebrare il bicentenario verdiano insieme all'Istituto italiano di cultura, mi sono soffermato sul palcoscenico vuoto del Teatro de São Carlos per cercare nel silenzio della sala l'eco improbabile di quella rappresentazione ormai mandata a memoria grazie ai dischi scoperti in età adolescenziale. Nei pochi giorni di permanenza in città, la programmazione stabilita dal teatro per la Trilogia verdiana mi ha consentito di vedere soltanto Rigoletto e Il trovatore, cosicché anche per me La traviata di Lisbona è destinata a rimanere idealmente per sempre non già quella (non vista) del 2013, bensì quella (non vista ma amata) del 1958.

Girando in quei giorni oziosamente per i vicoli del Bairro Alto mi sono imbattuto in una Livraria Antiquária che ha attirato la mia attenzione: più che libri, vecchi giornali e vario materiale usato. Entro; chiedo: "Tem livros sobre can- 
tores de ópera?”. Mi hanno portato verso uno scaffale in cui si affastellavano i programmi di sala delle stagioni d'opera al São Carlos negli anni '50-'60, evidente lascito di un appassionato melomane defunto: quanti titoli operistici in quelle ricchissime stagioni, con cast vocali oggi considerati insuperabili! Ma La traviata del marzo 1958 mancava clamorosamente all'appello fra quei tanti libretti consunti dal tempo... E sperai allora vivamente in cuor mio che l'anonimo spettatore lisbonese di cui ammiravo la collezione non si fosse perso a suo tempo l'ineffabile spettacolo oggetto di tanto culto, e che il relativo programma di sala fosse stato prelevato dalla collezione ad opera di qualche altro sognatore che si aggirava attorno al teatro, come me, alla ricerca del tempo perduto... 